\title{
GAMIFICACIÓN EN EL AULA DE EDUCACIÓN INFANTIL: UN PROYECTO PARA AUMENTAR LA SEGURIDAD EN EL ALUMNADO A TRAVÉS DE LA SUPERACIÓN DE RETOS
}

\author{
GAMIFICATION IN PRE-SCHOOL EDUCATION: \\ A PROJECT TO INCREASE SELF-CONFIDENCE AMONG \\ STUDENTS BY OVERCOMING CHALLENGES
}

\begin{abstract}
Andrea Romero Rodríguez ${ }^{a^{*}}$ y Javier Espinosa Gallardo
\end{abstract}
Fechas de recepción y aceptación: 18 de octubre de 2019, 4 de diciembre de 2019

Resumen: Es en la etapa de Educación Infantil cuando se produce una introducción al proceso de lectoescritura; de este modo, es cuando pueden observarse las mayores diferencias en el ritmo de aprendizaje entre unos y otros, lo que puede provocar una falta de confianza a la hora de desempeñar sus actividades. Por ello se ha pensado en una forma atractiva que estuviera basada en la superación de retos y que a su vez permitiera fomentar la motivación de nuestro alumnado durante todo el proceso de enseñanza-aprendizaje. De esta forma, se ha realizado una investigación bibliográfica sobre la gamificación como herramienta didáctica para desarrollar una mentalidad de crecimiento en el alumnado a través de un aprendizaje basado en retos (challenge-based learning), en un entorno en el que el juego es el protagonista. Este proyecto pretende unificar la seguridad en el alumnado y la gamificación, considerando que la oportuna conexión que existe entre ambas puede mejorar la necesidad de la que surge la creación de este proyecto.

Palabras clave: gamificación, trabajo cooperativo, Educación Infantil, motivación.

Abstract: It is in Pre-School Education when students are introduced in the process of reading and writing, and so it is also when the greatest differences between pupils in the learning pace are noticed. This leads to a lack of confidence when performing their activities.

${ }^{a}$ Maestra en Educación Infantil.

${ }^{*}$ Correspondencia: Andrea Romero Rodríguez. Calle Actor José Crespo, 1, 5. ${ }^{\circ}$ B 30002 Murcia. España.

E-mail: andrearr94@hotmail.com

${ }^{\mathrm{b}}$ Responsable de proyectos en la empresa NIUCO. Universidad Rey Juan Carlos I. 
Because of that, an attractive method has been designed, based on overcoming challenges that arise, which in turn allows to encourage the motivation of our students throughout their teaching-learning process. To this end, these authors have carried out a bibliographical research focusing on gamification as a didactic tool that can help develop students' personal growth by means of a challenge-based learning methodology in an environment where games are the protagonist. The aim of this project is to unify confidence and gamification, believing that their existing connection can help improve what this project addresses precisely.

Keywords: gamification, cooperative work, Pre-School Education, motivation.

\section{JUSTIFICACIÓN}

El principal objetivo que todo maestro de Educación Infantil debe marcarse es formar a niños y niñas seguros de sí mismos, y es de esta idea de la que se partirá durante la realización de todo el proyecto.

De este modo, se considera oportuna la puesta en marcha de un proyecto en el que se trabaje la superación del miedo a equivocarse y la frustración de manera interrelacionada. Para ello, se debe pensar en un hilo conductor que sea lo suficientemente atractivo y motivador para el alumnado.

El juego es uno de los principios generales que se establecen en la Orden ECI/3960/2007, de 19 de diciembre (BOE núm. 5, sábado 5 de enero 2008), por la que se establece el currículo y se regula la ordenación de la Educación Infantil, concretamente en el punto número 4 del artículo 2, el cual nos dice que los métodos de trabajo en ambos ciclos se basarán en las experiencias, en la actividad infantil y en el juego, y se aplicarán en un ambiente de seguridad, afecto y confianza para potenciar la autoestima y la integración social.

Partiendo de lo anterior, y sabiendo que la gamificación es una tendencia que avanza en la actualidad, esta podría utilizarse como herramienta didáctica para este propósito. Hablar de gamificación es hablar de motivación a través del entretenimiento y divertimento que aporta el juego por sí mismo (Contretas y Eguia, 2016). La gamificación afecta a nuestra faceta competitiva, de manera que nos permite ser capaces de analizar nuestras fortalezas, pero también nuestras debilidades, todo ello en un entorno de estimulación, pudiendo cometer errores y aprendiendo a través de la práctica (Fandos y González citados en Igado, 2013). 
Además, por medio de la gamificación el alumnado será capaz de asumir el error como algo natural, incidiendo en nuestra faceta competitiva, pudiendo analizar nuestras fortalezas y debilidades y entendiendo el error como una manera de aprender, consiguiendo así acabar con ese miedo a equivocarse del que parte este proyecto (Fandos y González, citados en Igado, 2013).

\section{FundAMENTACIÓN TEÓRICA}

\subsection{Introducción a la gamificación}

Existen diferentes autores que han dado diversas definiciones acerca de la gamificación, por ejemplo, Werbach y Hunter (2014) definen la gamificación como "uso de elementos de los juegos y técnicas del diseño de juegos en contextos que no son de juegos".

Borrás (2015) realiza una distinción entre los términos juego y jugar que es interesante tener en cuenta. Cuando se habla de juego nos referimos a una estructura cerrada, consiste en un sistema explícito de reglas que sirven de guía para los usuarios hacia metas discretas y resultados. El juego se encuentra aislado del mundo real. La gamificación lo que pretende es que exista una involucración con ese círculo. Por su parte, jugar consiste en la libertad que se da dentro de unos límites, basándose en disfrutar de la acción en sí misma que se está realizando, de divertirse.

Para llegar a terminar de comprender en qué consiste la gamificación sería conveniente diferenciar entre juego y actividad gamificada. Para ello, algunos autores ayudan a comprender la diferencia entre ambos términos. Por su parte, Foncubierta y Rodríguez (2014) reconocen el juego como algo concreto, mientras que la gamificación está basada en contenidos didácticos, es decir, se usan los elementos y pensamientos del juego para aderezar las actividades que se proponen. Además, añaden que cuando se plantean juegos en el aula entra en acción la fantasía, desarrollándose dicho juego en un espacio mágico de entretenimiento y diversión. Sin embargo, cuando se incorporan puntuaciones, resolución de retos o enigmas o una cuenta atrás lo que se pretende es estructurar el juego con unas reglas y unas pautas concretas. 
La clave reside en que a través de una actividad gamificada los alumnos no aprenden a través de juegos específicos, sino que aprenden como si estuvieran jugando a un juego. El juego tiene una serie de objetivos didácticos claros y marcados, es un juego con premeditación (Simöes, Díaz y Fernández, 2013).

Partiendo de todo lo anterior, ¿qué razones tenemos para gamificar las actividades en el aula?

\subsection{Razones por las que gamificar}

Autores como Oriol Borrás (2015), en su obra Fundamentos para la gamificación, hacen una clasificación de las razones por las cuales utilizar gamificación en el aula.

Uno de los principales motivos por los que es beneficioso gamificar en el aula es porque de este modo se activa la motivación por el aprendizaje. Gibson (2012) explica que a la gente por lo general le gusta jugar, pero que en muchas ocasiones las actividades de juego están carentes de motivación. De este modo, la gamificación introduce la motivación en las actividades, y en este caso concreto en la educación.

Por otra parte, es importante destacar la retroalimentación constante. Los participantes son jugadores, y por lo tanto son los principales protagonistas, sintiéndose involucrados, tomando sus propias decisiones, progresando y viendo como sus logros son reconocidos.

Además, a través de la gamificación el aprendizaje es más significativo, lo que permite mayor retención en la memoria, al hacer el contenido más atractivo. La gamificación parte de algo que llama la atención de los alumnos, que los estimula, y, por lo tanto, ayuda a que genere dicho aprendizaje durante todo el proceso.

Por otro lado, los alumnos van a ir ganando en autonomía. La gamificación consiste en el planteamiento de retos que se han de superar, existiendo una progresión en el desempeño de las actividades, de manera que a lo largo de esta progresión también es mejor en cuanto al grado de conocimiento y de autonomía en las diferentes habilidades y actividades (Igado, 2013).

Posada (2017) nos habla de la alfabetización digital como otra de las razones por la cual es conveniente la inversión de esta en las aulas, tratándose 
de la aplicación de las mecánicas de juegos a las tareas escolares usando las TIC, ya que así sería posible la mejora de las competencias, el incremento de la motivación y la participación del alumnado.

\subsection{Aprendizaje basado en retos}

Para trabajar motivando al alumnado y así poder alcanzar de manera más efectiva los objetivos propuestos, se hará uso del challenge-based learning (CBL), o lo que es lo mismo, aprendizaje basado en retos (ABR); cómo aprender a través de una temática común, planteando distintos retos relativos a dicho tema que el alumnado debe superar. Los retos que se plantean llevan consigo el aporte de distintas soluciones concretas de las que pueden verse beneficiados como parte de la sociedad, para lo que se hace uso de herramientas tecnológicas, recursos internos y externos y, por supuesto, del profesorado que los guíe (Cordray, Harris y Klein, citado en Fidalgo, Sein-Echaluce y Klein, 2016).

Según el Informe EduTrens del Observatorio de Innovación Educativa del Tecnológico de Monterey (información ampliable en https://observatorio. itesm.mx/edutrendsabr/), el CBL está inspirado en la búsqueda de soluciones a desafíos globales y originados en contextos externos a la formación. En cambio, su proceso de aprendizaje y su forma de puesta en práctica están basados en los principios del aprendizaje activo, como el aprendizaje basado en problemas, el aprendizaje basado en proyectos o el vivencial, integrando elementos de investigación e interdisciplinaridad, girando todo en torno al alumnado.

Según este mismo informe, también podemos enumerar distintos puntos a favor del CBL, entre los que se encuentran:

- El alumnado alcanza una comprensión más profunda de los temas, así como el desarrollo de su creatividad.

- Involucración por parte del alumnado.

- Mayor sensibilidad ante la situación que se da, desarrollando procesos de investigación.

- Se alcanza un aprendizaje más significativo, estableciéndose una relación entre lo académico y el mundo que le rodea.

- Desarrollo de habilidades comunicativas a través de técnicas sociales. 


\subsection{Principios psicológicos de la gamificación}

En esta investigación pretendemos hacer uso de la gamificación como un medio de aprendizaje; de este modo, es importante tener en cuenta la manera en la que se pretende que este aprendizaje ocurra.

A continuación, hablaremos de dos importantes teorías del aprendizaje que deben estar presentes en cualquier propuesta didáctica gamificada que quiera ser puesta en práctica exitosamente.

\section{Conductismo}

El conductismo se refiere al aprendizaje como los cambios que se producen en una conducta observable, en relación con la forma o la frecuencia de esas conductas. Para este modelo, el aprendizaje se da o se demuestra cuando una respuesta es correcta o apropiada después de la presentación de un estímulo exterior específico. De este modo, el conductismo centra la atención en las consecuencias que tienen las conductas y afirma que las respuestas que vienen seguidas de refuerzos son más probables que se repitan en el futuro (Ertmer y Newby, 1993).

Por lo tanto, podemos decir que habrá tres aspectos que estarán siempre presentes y que debemos extrapolar a la gamificación:

- Observación: cómo están llevado a cabo la acción los alumnos.

- Retroalimentación: lo que se recibe por parte de la persona que observa.

- Refuerzo: el aprendizaje se produce cuando la acción o conducta va seguida de un refuerzo.

\section{Cognitivismo}

Para los cognitivistas el aprendizaje se asemeja a cambios discretos entre los estados del conocimiento más que a los cambios en la probabilidad de respuesta. Las teorías cognitivas se encargan de la conceptualización de los procesos de aprendizaje del alumnado y se ocupan de conocer de qué manera la información es recibida, almacenada, organizada y localizada. De este modo, siguiendo esta teoría, el aprendizaje tiene relación con qué es lo que 
conocen y cómo adquieren dicho conocimiento (Jonassen citado en Ertmer y Newby, 1993). De este modo, teniendo en consideración las teorías cognitivas para la aplicación de la gamificación en las aulas, es importante hablar de las recompensas, las cuales tienen un papel protagonista durante cualquier proyecto gamificado. Según Borrás (2015) es necesario hacer una distinción entre las distintas recompensas que pueden darse y de qué forma tenerlas en cuenta a la hora de gamificar siguiendo las teorías cognitivas. Existen recompensas intrínsecas, a través de las cuales el alumno prestaría atención a las consecuencias, es decir, actuaría por la acción en sí misma. Por otro lado, es necesario hablar de recompensas extrínsecas, en las que el alumnado actuaría por una recompensa externa.

\subsection{El juego de gamificar}

Para comenzar, debemos partir de los elementos del juego de los que haremos uso, conociendo como elementos del juego aquellas herramientas que se crean y se ponen en práctica para incrementar la motivación, proveer estatus y ver al jugador dentro de una plataforma concreta, lo que hace posible que este esté involucrado en dicha experiencia (Aguilera, Fúquena y Ríos, 2014).

Por su parte, Herranz (2013) señala los siguientes elementos:

\subsubsection{Dinámicas}

Las dinámicas consisten en los aspectos globales en los que un sistema gamificado está orientado y tienen una relación directa con los efectos, motivaciones y deseos que se pretenden proporcionar en el jugador (Herranz, 2013).

Según el Informe Edutrens Gamificación (2016) del Observatorio de Innovación Educativa del Tecnológico de Monterrey, podemos distinguir los siguientes elementos dentro de las dinámicas:

- Reglas: dentro de las reglas podemos distinguir las restricciones del juego, la asignación de turnos, permanecer con vida, ganar o perder puntos o alcanzar un objetivo. 
- Narrativa: son los avatares, identidades, personajes, mundos o ambientes que forman parte del juego.

- Sorpresa: consiste en las recompensas aleatorias que van recibiendo durante el juego, por ejemplo, los huevos de pascua. Consiste en motivar a los jugadores mediante elementos inesperados, con el fin de mantener al jugador involucrado.

\subsubsection{Mecánicas}

Las mecánicas son las reglas que intentan que los juegos que se realizan se puedan disfrutar, que generen cierto compromiso, ofreciéndoles retos y un camino que seguir (Cortizo, Carrero, Monsalve, Velasco y Díaz del Dedo, 2011).

Para la clasificación de las distintas mecánicas seguimos basándonos en Informe Edutrens Gamification (2016), que se ha citado anteriormente. Las mecánicas son las siguientes:

- Metas y objetivos: son desafíos, misiones y retos. Presentan un reto o un problema que hay que resolver y sirven para generar motivación.

- Libertad de elegir: son las rutas para llegar a la meta, posibles privilegios y poderes. De este modo, el jugador tiene la libertad de elegir distintas posibilidades para avanzar.

- Libertad para equivocarse: consiste en que a través de las vidas, puntos de restauración y tentativas los jugadores sean capaces de experimentar, sin miedo a equivocarse, el ensayo-error como estrategia de aprendizaje.

- Recompensas: vidas, monedas virtuales, equipamiento, poderes... Consiste en acercarse a través de estas recompensas a alcanzar los objetivos del juego.

- Retroalimentación: la retroalimentación se realiza a través de barras de proceso, iconos de acierto y error, insignias... Estas deberían ofrecerse de manera inmediata, indicando que se está acercando a conseguir el objetivo. 


\subsubsection{Componentes del juego}

Los componentes son la forma físicamente más específica que adquieren las dinámicas y mecánicas. Consiste en aquellos elementos en los que estas se apoyan para que se desarrollen los comportamientos deseados en los jugadores (González, 2016).

FIGURA 1

Elementos del juego. Creación propia

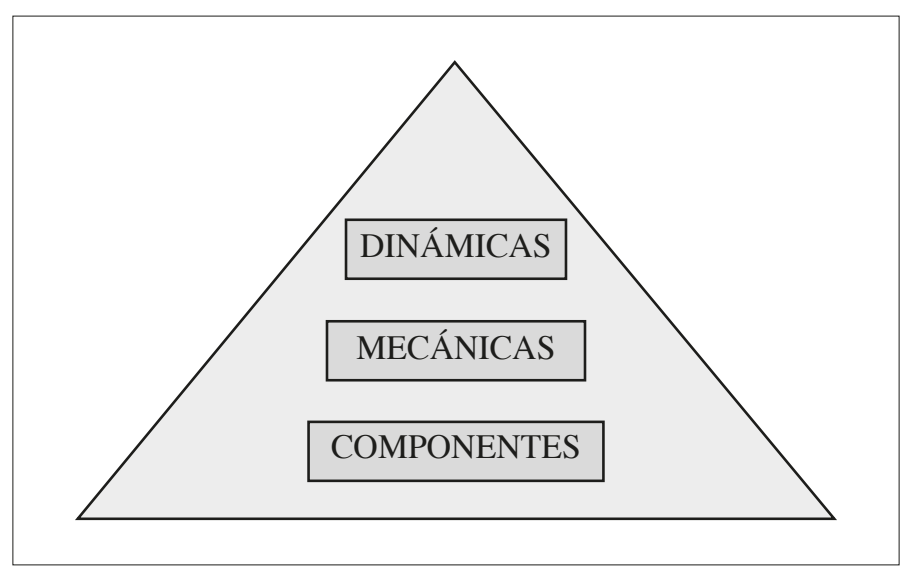

\subsection{Tipos de jugadores y gamificación}

Para poder poner en práctica un proyecto gamificado de manera satisfactoria es necesario conocer los tipos de jugadores que existen dentro de la gamificación. Para ello, nos basaremos en la clasificiación de Bartle y Marczewski (citado en Contreras y Eguia, 2016). De este modo, podemos identificar los siguientes tipos:

- Socializadores: están motivados por las relaciones sociales, su intención es interactuar y promover conexiones sociales con los otros.

- Filántropos: están motivados por el objetivo y el significado de las acciones, quieren ayudar, dar y prosperar la vida de los demás, sin esperar nada a cambio. 
- Exploradores: la independencia y la autonomía son los protagonistas, quieren explorar, innovar y crear.

- Triunfadores: la maestría tiene un papel fundamental, quieren aprender cosas nuevas y mejorar en el transcurso del juego.

- Jugadores: están alentados por las recompensas y luchan por conseguir dichos reconocimientos.

- Revolucionarios: están incentivados por el cambio, buscan la alteración del sistema por sí mismos o a través de otros para generar cambios positivos o negativos.

Pero, partiendo de esto, podemos conocer distintos tipos de gamificación, ¿cuáles podemos destacar?

Werbach y Hunter (citado en Borrás, 2015) proponen tres tipos de gamificación:

- Interna: Se trata de mejorar la motivación dentro de una organización.

- Externa: Se tiene como objetivo la involucración de los participantes a través de la mejora de las relaciones que se establezcan entre ellos, de manera individual y como conjunto.

- Cambio de comportamiento: Consiste en tratar de aumentar el aprendizaje mientras se disfruta, generando nuevos hábitos en los jugadores, y conseguir que escojan opciones más convenientes para rediseñar la clase.

\section{Metodología}

Anteriormente al diseño de cualquier proyecto gamificado debemos tener en cuenta una serie de principios metodológicos propios de la gamificación para que el proyecto pueda llevarse a cabo de forma exitosa, siendo capaces de adaptar los principios propios de un videojuego al proceso enseñanzaaprendizaje, de manera que se pueda alcanzar el logro de competencias tanto individuales como colectivas, al tiempo que se fomenta la colaboración entre el alumnado. Para ello, nos basaremos en la clasificación que han realizado por Barragán, Ceada, Andújar, Irigoyen, Gómez y Artaza (2015). 
De este modo, las características propias de los videojuegos que debemos adaptar al proceso enseñanza-aprendizaje son las siguientes:

1. Jugabilidad: Consiste en la manera de manejar el juego. Para que el juego llame y capte la atención de los niños debe ser fácil e intuitivo, de este modo el aprendizaje puede ser costoso y no obtener la respuesta esperada en el alumnado.

2. Colaboración entre los jugadores: El juego tiene como objetivo la consecución de los objetivos de manera cooperativa, por lo que al tener la presencia de más de un jugador al mismo tiempo la cooperación debe estar siempre presente de algún modo.

3. Asumir un papel durante el juego: El alumno pasa de actuar como tal a actuar como un jugador, por lo que sus metas y procedimientos ahora son diferentes. El papel del jugador va evolucionando a medida que asume los distintos logros y supera los diferentes retos.

4. Retroalimentación: Esta debe estar presente durante todo el desarrollo del juego, especialmente en los avances más significativos que se vayan asumiendo. Del mismo modo, deben ser lo suficientemente evidentes para que el alumno sea lo suficientemente consciente.

5. Engagement: Consiste en el grado de compromiso que adquiere el jugador, es decir, el grado de participación, motivación y actividad que demuestra durante el juego.

6. Diversión: La diversión debe ser la protagonista durante todo el proceso de enseñanza-aprendizaje de un proyecto gamificado. De este modo, el docente será capaz de captar la atención del alumnado y lograr un aprendizaje más significativo.

\section{DiseÑo DEL PROYECTO}

El siguiente proyecto gamificado, y por tanto las actividades programadas para este, se llevarán a cabo en una clase del segundo curso del segundo ciclo de Educación Infantil, teniendo en cuenta el principal objetivo del proyecto, fomentar la seguridad y confianza en uno mismo en el aula. En esta etapa educativa se empieza con el proceso de lectura, en el que los niños empiezan 
a leer sus primeras palabras, por lo que se pueden observar diferentes ritmos de aprendizaje entre ellos, lo que en ocasiones puede generar grandes inseguridades en aquellos que ven que no están al mismo nivel que el resto. De este modo, se genera en algunos niños el conocido "miedo a equivocarse", que provoca que aquellos más inseguros tengan miedo a expresar lo que piensan sobre algún tema en concreto, a contestar una pregunta o incluso a enfrentarse a ciertos conflictos de la vida diaria.

La fundamentación metodológica en la que estarán basados los retos puede verse reflejada en la figura 2 , en la que se aprecia el objetivo general que se debe alcanzar, el cual está rodeado de los elementos necesarios para lograrlo, que en este caso corresponden a los niveles en los que se divide el siguiente proyecto gamificado, a través de los cuales estarán agrupados los siguientes retos.

FIGURA 2

Niveles del proyecto gamificado. Creación propia

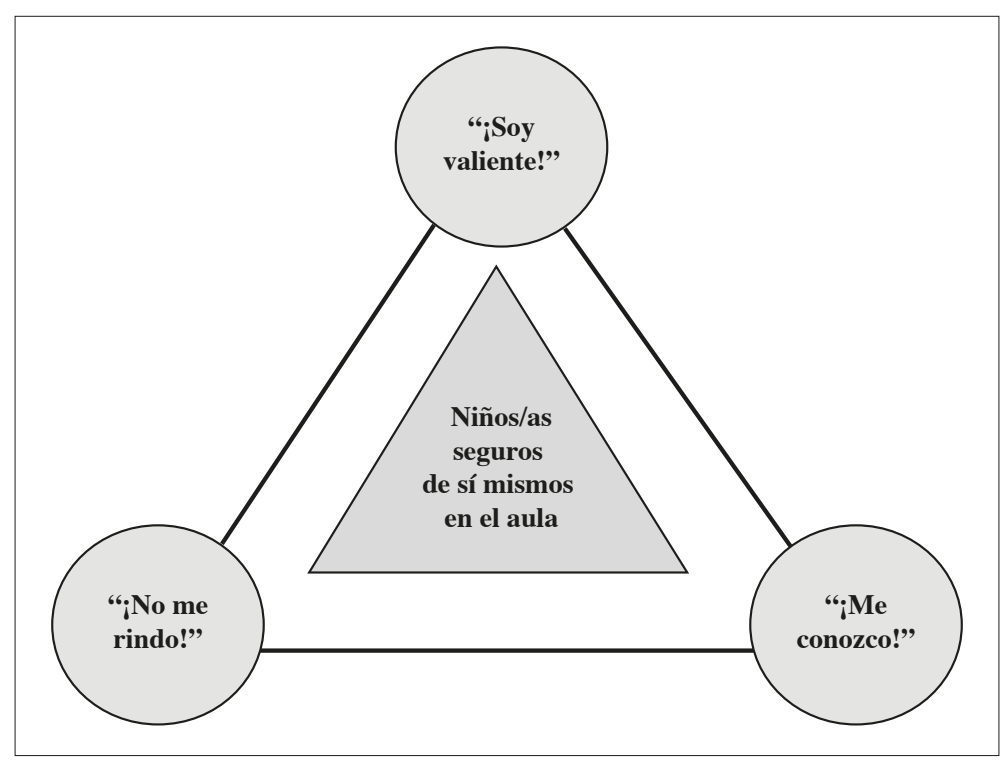




\subsection{Contenidos}

A través del presente proyecto gamificado se trabajarán distintos contenidos durante el desempeño de los diferentes retos que se plantean posteriormente. Estos contenidos están directamente relacionados con los objetivos de los que surge esta propuesta metodológica. Los contenidos que hay que trabajar son los siguientes:

- Reconocimiento de la valentía.

- Identificación de situaciones a las que tenga que hacer frente con valentía.

- Superación de obstáculos que se encuentren en el desempeño de las tareas.

- Disposición persistente que ayude a no rendirse.

- Reconocimiento de las propias fortalezas y debilidades.

- Identificación de las mejoras experimentadas con el paso del tiempo.

- Utilización de las nuevas tecnologías como elemento motivador del aprendizaje.

- Cooperación a través del trabajo en equipo.

\subsection{Dinámicas: narrativa, equipos, avatares y puntos}

En todo proyecto gamificado es necesaria una narrativa que motive al alumnado. De este modo, usaremos la historia del Mago de Oz. Los niños se convertirán en los protagonistas de la historia, de manera que a través de la superación de los distintos retos que se proponen conseguirán llegar hasta la Ciudad Esmeralda, donde podrán pedirle al Mago de Oz que les dé la confianza que necesiten.

A medida que los equipos vayan alcanzando los retos que podremos ver a continuación irán sumando puntos en la aplicación de ClassDojo, donde podrán verse los distintos equipos con la foto del nombre del equipo correspondiente, así como los puntos que pueden recibir según las habilidades empleadas en la tarea. ClassDojo es una herramienta a través de la cual podemos realizar un seguimiento de las actitudes de aprendizaje, ofreciendo una 
interfaz abierta, atractiva y fácil de usar. Además, es personalizable según las necesidades que se tengan en ese momento, pudiéndose ajustar a la temática, el tipo de agrupación, etc.

\subsection{Mecánicas: Retos}

A continuación, se indican a modo de ejemplo algunas de las actividades que formarán parte de cada uno de los niveles mencionados anteriormente en el diseño del proyecto.

Nivel 1: "Soy valiente"

\section{"La caja que los miedos atrapa"}

Motivación inicial vinculada a la narrativa: Se hará uso en un primer lugar del cuento del León Cobarde, uno de los protagonistas del Mago de Oz, el cual, a pesar de ser el rey de la selva, muestra sus miedos y le pide al Mago de Oz que le dé el valor suficiente para hacer frente a ellos, sin ser consciente de que este valor lo tiene en su interior. De este modo, es oportuno el uso de este recurso al inicio del proyecto, ya que a través de esta historia los niños pueden ser conscientes de que todos tienen miedos pero que deben hacerles frente a través de la valentía, la cual está presente en todos ellos, solo tienen que querer mostrarla.

Acción: Después de la lectura de este cuento, en asamblea, se establecerá un debate en el cual se hablará de los miedos, diciendo cada uno algo que le provoca miedo, para finalmente plasmarlo en un dibujo que se meterá en la "caja que atrapa los miedos", la cual estará presente en el aula durante todo el proyecto, pudiendo hacer uso de ella para actividades posteriores.

Retroalimentación: La recompensa que obtendrán será la caja sin decorar para que, por equipos, se encarguen de decorarla. Una vez obtenida la caja y decorada sumarán puntos en ClassDojo. 
Nivel 2: "No me rindo"

"Baldosas amarillas hacia nuestras metas"

Motivación inicial vinculada a la narrativa: Recibimos respuesta del Mago de Oz, y nos indica el camino para llegar a él. Se trata de un camino de baldosas amarillas. El mago les indicó que para ser unos niños seguros de sí mismos, además de adquirir valentía deberían superar metas, para lo que necesitarían persistencia. De este modo, les indicó que durante el camino de baldosas amarillas tendría que marcarse una meta personal que dividiría el camino en cuatro ("ya salgo", "me voy esforzando", "casi lo tengo" y "iconseguido!").

Acción: Cada niño tendrá un camino de baldosas que tendrán que colorear como la que el mago les envió, es decir, de color amarillo. Cuando lo tengan, se establecerá en la asamblea una sesión dedicada a hablar sobre aquellas cosas en las que pueden mejorar, estableciendo una meta para cada niño que la profesora escribirá en un papel. A continuación, tendrán que pegar su meta a una foto suya, y empezarán el recorrido por el camino de baldosas amarillas ("ya salgo") que llegará a la Ciudad de Esmeralda ("conseguido") si son capaces de lograr sus metas. Las metas estarán puestas en un mural con los caminos de baldosas amarillas de cada uno, de manera que puedan ser conscientes de sus avances.

Retroalimentación: Llegar a la Ciudad de Esmeralda y conseguir un punto en ClassDojo.

Nivel 3: "Me conozco"

"Nos conocemos con códigos QR"

Motivación inicial vinculada a la narrativa: Por último, para recibir la ayuda del Mago de Oz necesitaremos superar un último reto. El mago nos manda un mensaje en el que nos dice que quiere conocernos; para ello nos deja en el aula distintos códigos QR que tendremos que descubrir y una ficha para realizar en Edmodo con sus familias. 
Acción: A través de este reto los alumnos tendrán que descubrir los cambios que han ido experimentando con el paso del tiempo. Para ello, empezarán descubriendo los cambios físicos descifrando códigos QR. Los alumnos tendrán un código QR por cada uno de los niños, cada código esconde una foto de cada uno de los niños cuando era bebé. Los niños se colocarán por equipos y se dividirán los códigos $\mathrm{QR}$ en cada uno de los equipos. Los equipos tendrán que descifrar los códigos QR con la tableta del aula y al descubrir la imagen tendrán que buscarla por la clase. Cuando todos los equipos tengan todas las fotos tendrán que debatir sobre a quién corresponde esa foto de bebé. Al final de la sesión los niños tendrán que decir si la foto que le han asignado corresponde a ellos o no. A continuación, cada niño tendrá que llevarse esa foto a casa para realizar una actividad con sus familias en casa. En esta actividad se va llevar a cabo un recorrido por la vida de los niños, y quién mejor para ayudarlos a hacerlo que sus familias. Para ello utilizaremos la herramienta Edmodo. Le daremos a las familias una ficha, la cual tendrán que rellenar de manera visual (fotos, dibujos...) con información que describa a sus hijos en el pasado (“Así era"), en el presente (“Así soy”) y en el futuro ("Así me gustaría ser"). De modo que lo que se pretende es analizar las mejoras y los cambios que han ido experimentado los niños a lo largo del paso del tiempo, desde cambios físicos a mejoras en el comportamiento, pudiendo hacer un análisis de aquellas cosas que antes no sabía hacer y ahora sí, etc. Además, esto servirá para que les ayude a saber cómo son en la realidad, conociendo sus fortalezas y debilidades, para así plantearse al final de la plantilla cómo les gustaría verse en un futuro. Una vez realizada la ficha del paso de tiempo con las familias tendrán que publicarlas en Edmodo (una herramienta de comunicación para compartir materiales) para explicarlas al resto de sus compañeros, de modo que se establecerá una sesión de asamblea para que los niños en gran grupo puedan ir contando cómo han cambiado a lo largo del tiempo. Al mismo tiempo, los compañeros con sus familias podrán comentar las fichas de los compañeros en Edmodo, existiendo así una comunicación entre los alumnos y las familias.

Retroalimentación: Al terminar este reto conseguiremos llegar a la Ciudad de Esmeralda y sumaremos punto en ClassDojo. 


\section{EVALUACIÓN DEL PROYECTO GAMIFICADO}

Debido a que la propuesta metodológica anteriormente presentada está basada en la superación de niveles a través de la resolución de distintos retos, es especialmente conveniente que su evaluación se realice mediante la teoría de la carga cognitiva de Sweller.

La teoría está basada en modelos de enseñanza explícitos, creyendo que a través de estos modelos el cerebro aprende de forma más efectiva. En este modelo se enseña a los alumnos qué hay que hacer y cómo hacerlo de forma efectiva (Kirschner, Sweller y Clark, 2006 citados en Centre for Education Statistics and Evaluation, 2014). Nuestro proyecto consiste en la superación de retos que los niños y las niñas tienen que superar para llegar a los niveles posteriores, dándoles las indicaciones a través de la narrativa de manera explícita, por lo que es óptimo hacer uso de esta teoría en la evaluación.

Se conocen diferentes cargas cognitivas según Paas, Tuovinen, Tabbers y Gerven (2003), las cuales veremos a continuación, así como también observaremos en qué medida están presentes en nuestra propuesta metodológica.

Carga cognitiva intrínseca: se trata de aquella carga que va unida a la complejidad de la tarea y al nivel de experiencia del alumno en este caso. Depende entonces de dos variables: la dificultad intrínseca del material que hay que aprender y el dominio del alumno. En este sentido, la información previa deberá tenerse en cuenta, pues los esquemas que ya están formados en la memoria de largo plazo influyen directamente en la capacidad de la memoria de trabajo del aprendiz. Una tarea específica puede ser compleja para un novato; no obstante, será sencilla para el experto (Chong, 2005 citado en Andrade-Lotero, 2012). Esto será tenido en cuenta a lo largo de toda nuestra metodología durante el diseño de los retos propuestos. Para poder evaluar de manera coherente al alumnado debemos considerar las experiencias previas de cada uno, de forma que se adecue a su nivel de conocimiento, que supondrá un reto real para ellos, y que conlleva cierta dificultad a la que tendrán que hacer frente.

Carga cognitiva extrínseca: está relacionada con aquella carga innecesaria y que puede provocar, por tanto, saturación, lo que afecta a la memoria de trabajo. Cuando el alumno está interactuando con un material o interfaz en cuyo diseño o ejecución existe la presencia de elementos irrelevantes, aumenta la 
carga extrínseca, que entorpece los procesos tanto de construcción como de automatización de esquemas. El mal diseño instruccional es aquel que no favorece el aprendizaje e introduce "ruido", por lo que el aprendiz utilizará sus limitados recursos cognitivos para poner atención a aspectos poco relevantes en la resolución de la tarea. Además, como la carga cognitiva es aditiva, las cargas intrínseca y extrínseca se suman, lo cual deja poco espacio para la carga relevante (Anthony, 2008, citado en Andrade-Lotero, 2012). A veces, este tipo de carga cognitiva está presente en las propuestas metodológicas que se llevan a cabo; por tanto, debemos intentar evitarlas. Esta propuesta se ha diseñado tratando de evitar sobrecargas que puedan alejar a los niños de la consecución de los objetivos marcados. No obstante, durante su desarrollo, podemos observar la presencia de elementos que no son lo suficientemente relevantes, de manera que se podrían suprimir durante la marcha.

Carga cognitiva relevante: es la directamente responsable de contribuir al aprendizaje. Se constituye a partir de procesos cognitivos adecuados, como las conceptualizaciones y las elaboraciones. Este tipo de carga está relacionada con el diseño de la interfaz, puesto que la manera como se presente la información y el tipo de actividades que se crean pueden favorecer el aprendizaje del individuo. Aunque la carga relevante también se suma a la carga cognitiva total, esta representa los recursos que se invierten directamente en el aprendizaje del material, como la construcción de esquemas (Artino, 2008 citado en Andrade-Lotero, 2012). Esta última carga cognitiva será la protagonista de la propuesta metodológica, y a través de ella evaluaremos la propuesta, los elementos y materiales que intervienen en los distintos retos para comprobar de qué manera contribuyen al aprendizaje, y, por tanto, en la generación de esquemas.

Para evaluar la presente propuesta metodológica es necesario tener en cuenta que la carga cognitiva de la que hablamos no puede ser medida de manera directa, así para ello es necesaria la medición de otras dimensiones que sí pueden ser medidas, como son la carga mental, el esfuerzo mental y el desempeño (Andrade-Lotero, 2012), como puede verse reflejado en la figura 3.

Según este mismo autor, la carga mental ocurre por la interacción que surge entre las características que tiene una tarea y las características del alumno. Las tareas pueden tener un mayor o menor grado de dificultad, del mismo modo que el alumno puede tener distintas aptitudes o habilidades según la 
tarea en cuestión. De este modo, la carga mental se puede medir mediante la evaluación de estas dos variables, lo cual demostraría cómo de apropiado es el alumnado para la resolución de una tarea determinada.

Por su parte, Hart (2006, citado en Andrade-Lotero, 2012) se refiere a que la evaluación del esfuerzo mental está relacionada con los recursos cognitivos que se emplean para la resolución de una tarea, reflejando la carga cognitiva actual. Este esfuerzo mental puede ser medido cuando los participantes están realizando la tarea.

El desempeño, según Paas, Tuovinen, Tabbers y Pascal Gerven (2003, citado en Andrade-Lotero, 2012), consiste en el desempeño de un aspecto de la carga cognitiva. Es entendido como logro cuantificable del alumno, por ejemplo, el número correcto de respuestas, la cantidad de errores o el tiempo que le llevó resolver la tarea, pudiendo ser medido mientras la gente está trabajando o inmediatamente después.

FIGURA 3

Representación gráfica de la evaluación del proyecto. Creación propia

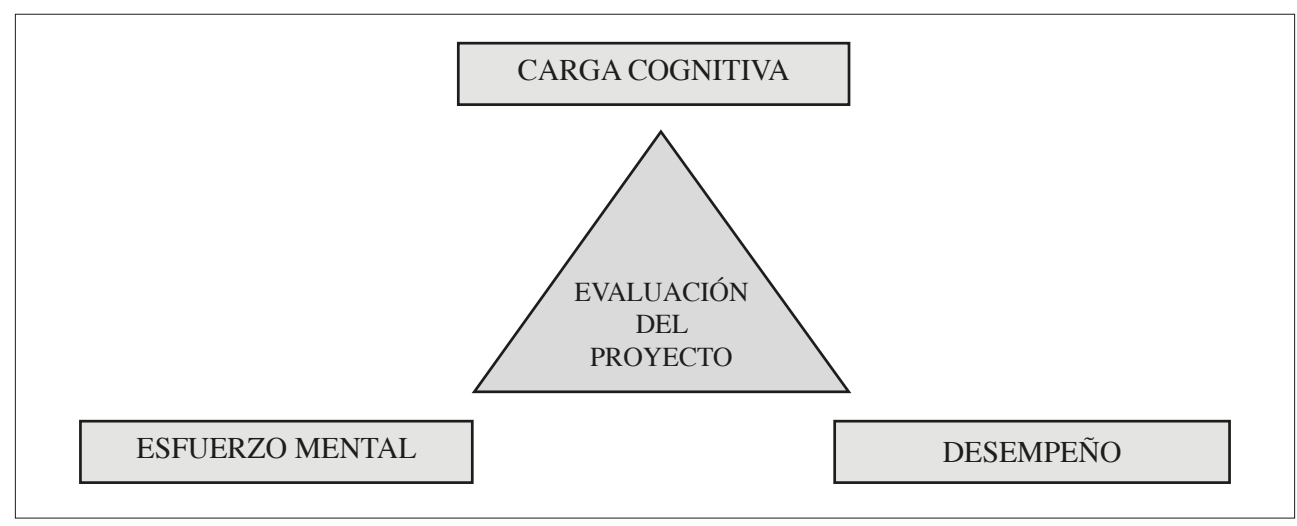

\section{Conclusiones}

Se ha partido de base de la importancia de formar alumnos seguros de sí mismos en las aulas desde la etapa de Educación Infantil, creyendo en la repercusión favorable que esto puede tener en su futuro, tanto personal como profesional y considerando que en las aulas pueden desarrollarse las mayores 
inseguridades, debido a las diferencias que en muchas ocasiones pueden darse entre el alumnado.

Por lo tanto, se pensó en un proyecto que pudiera trabajar esta necesidad y que al mismo tiempo fuera motivador para el alumnado; por ello se pensó en una herramienta como la gamificación para hacerlo. Las nuevas tecnologías están presentes en nuestra sociedad y forman parte de la mayoría de los niños, razón por la cual se creyó que este sería el elemento motivador principal durante el proceso enseñanza-aprendizaje.

A través de la gamificación se trabaja la resolución de retos y, por consiguiente, la superación de niveles. Estos niveles se pensaron para trabajar distintas fortalezas que favorecieran al desarrollo de una mayor seguridad en sí mismos, la valentía, la persistencia y el autoconcepto. A través de estos retos los niños podrán hacer frente al error, y ser capaces de, poco a poco, perder ese miedo a equivocarse que encontramos en las aulas en la actualidad.

Este proyecto puede estar contemplado dentro de la Acción Tutorial, la competencia digital y la educación en valores. Por otro lado, este sirve de ayuda para el alumnado, ayudándoles a trabajar la seguridad en uno mismo mediante la aplicación de la innovación tecnológica, por lo que creo en la posibilidad de la aplicación de este proyecto en las aulas.

\section{BiBLIOGRAFÍA}

Acosta, R. (2004). La autoestima en la educación. Límite: revista de filosofía y psicología 11, 82-95.

Andrade-Lotero, L. A. (2012). Teoría de la carga cognitiva, diseño multimedia y aprendizaje: un estado del arte. Magis, Revista Internacional de Investigación en Educación 5(10), 75-92.

Barragán, A. J., Ceada y Andúuar, J. M., Irigoyen, E., Gómez, V. y Artaza, F. (2015). Una propuesta para la motivación del alumnado de ingeniería mediante técnicas de gamificación. Actas de las XXXVI Jornadas de Automática, Bilbao.

Borrás, O. (2015). Fundamentos de la gamificación. Recuperado de: http:// oa.upm.es/35517/1/fundamentos $\% 20 \mathrm{de} \% 201 \mathrm{a} \% 20$ gamificacion_v1_1.pdf 
Contreras, R. S. y Eguia, J. (2016). Gamificación en aulas universitarias. Recuperado de: http://incom.uab.cat/download/eBook_incomuab_gamificacion.pdf.

Cortizo, J. C., Carrero, F. M., Monsalve, B., Velasco, A., Díaz del Dedo, L. I. y PÉrez, J. (2011). Gamificación y Docencia: Lo que la Universidad tiene que aprender de los Videojuegos. VIII Jornadas Internacionales de Innovación Universitaria. Retos y oportunidades del desarrollo de los nuevos títulos en educación superior, Murcia.

Cosentino, A. C. (2010). Evaluación de las virtudes y fortalezas humanas en población de habla hispana. Psicodebate 10, 53-62.

Díaz, J. y Troyano, Y. (2013). El potencial de la gamificación aplicado al ámbito educativo. Innovación Educativa: respuesta en tiempos de incertidumbre. III Jornadas de Innovación Docente, Sevilla.

Ertmer, P. y Newby, T. (1993). Conductismo, cognitivismo y constructivismo: una comparación de los aspectos críticos desde la perspectiva del diseño de instrucción. Performance improvement quarterly 6(4), 50-72.

Fidalgo, Á., García, F. J. y Sein-Echaluce, M. L. (2017). Aprendizaje Basado en Retos en una asignatura académica universitaria. Revista Iberoamericana de Informática Educativa 25, 1-8.

Foncubierta, J. y Rodríguez, C. (2015). Didáctica de la gamificación en la clase de español. Programa de Desarrollo Profesional. Madrid: Editorial Edinumen.

Gallardo, M. H. Gamificar: el uso de los elementos del juego en la enseñanza del español.

Gallego, F. J., Molina, R. y Llorens, F. (2014). Gamificar una propuesta docente. Diseñando experiencias positivas de aprendizaje. XX Jornadas sobre la enseñanza universitaria de la informática, Alicante.

GiBson, R. (2011, 12 de enero). Show gamification some love. $M C V$. Recuperado de: https://www.mcvuk.com/development/show-gamification-somelove.

GonZÁLEZ, J. (2016). Gamificación: hagamos que aprender sea divertido (trabajo fin de máster). Navarra: Universidad Pública de Navarra.

González-Pineda, J. A., NúÑez, J. C., GonzÁlez-Pumariega, S. G y García, M. S. (1997). Autoconcepto, autoestima y aprendizaje escolar. Psicothema 9(2), 271-289. 
GrinhauZ, A. S. (2012). El análisis de las virtudes y fortalezas en niños: una breve revisión teórica. Psicodebate 12, 39-61.

Herranz, E. (2013, febrero). Gamification, I Feria Informática, Madrid: Universidad Carlos III.

IGADO, M. F. (2013). La gamificación como motivación en los entornos virtuales de aprendizaje. Ludoteracy, creación colectiva y aprendizaje. II Congreso internacional Educación Mediática y Competencia Digital, La Rioja.

NARANJo, M. L. (2007). Autoestima: un factor relevante en la vida de la persona y tema esencial del proceso educativo. Revista Electrónica Actualidades Investigativas en Educación 7(3), 1-27.

O’SHEa, C. (2006). En busca de los valores. Coherencia, fidelidad, generosidad, valentía... Una apuesta para una vida con sentido. Madrid: La esfera de los libros.

Palomera, R. (2009). Educando para la felicidad, en Fernández-Abascal (coord.), Emociones positivas (pp. 247-276). Madrid: Pirámide.

Paas, F., Tuovinen, J. E., Tabbers, H. y Van Gerven, P. W. (2003). Cognitive load measurement as a means to advance cognitive load theory. Educational psychologist 38(1), 63-71.

PosAdA, F. (2017). Gamifica tu aula: experiencia de gamificación TIC para el aula. CIVE: Actas del V Congreso Internacional de Videojuegos y Educación, Las Palmas.

Ramírez, J. L. (2014). Gamificación. Mecánicas de juegos en tu vida personal y profesional. SC Libro.

SimÖes, J., DíAz, R. y FernÁndez, A. (2013). A social gamification framework for a K-6 learning platform. Computers in Human Behavior, Elsevier 29(2), 345-353.

Tecnológico de Monterrey (2015). Reporte Edu Trends. Aprendizaje basado en retos. Recuperado de: https://observatorio.itesm.mx/edutrendsabr/.

Tecnológico de Monterrey (2016). Reporte Edu Trends. Gamificación. Recuperado de: https://observatorio.itesm.mx/edutrendsgamificacion/.

Zichermann, G. y Cunningham, C. (2011). Gamification by Design: Implementing Game Mechanics in Web and Mobile Apps. Cambridge: O'Reilly Media. 